\title{
EVALUATION OF THE EFFECTIVENESS OF TRANSCUTANEOUS BILIRUBINOMETRY IN REDUCING THE NUMBER OF SERUM BILIRUBIN TESTS IN NEONATAL JAUNDICE
}

\author{
D.J. Lee ${ }^{1}$, F. Gohar ${ }^{1}$, P. Newland ${ }^{2}$, C.W. Yoxall ${ }^{3}$ \\ ${ }^{l}$ Neonatal Intensive Care Unit, Liverpool Women's Hospital, ${ }^{2}$ Principle Biochemist, Biochemistry, Royal \\ Liverpool Children's Hospital, ${ }^{3}$ Consultant Neonatologist, Neonatal Intensive Care Unit, Liverpool Women's \\ Hospital, Liverpool, UK
}

Background: Bilicheck is a non-invasive device that uses multiwavelength spectral reflectance analysis to measure bilirubin levels transcutaneously. Previous work in our institution predicted that using bilicheck in jaundiced babies born after 34 weeks may reduce the number of serum bilirubin tests (SBR's) needed by up to $55 \%$. On the basis of this work, the use of Bilicheck was introduced into clinical practise in 2003.

Aim: To determine whether clinical use of Bilicheck has resulted in a reduction of SBR's in clinical practice and quantify that reduction.

Methods: A retrospective evaluation of all SBR's taken in 2002 (pre Bilicheck) and 2009 (post Bilicheck) compared the frequency of blood sampling for bilirubin estimation and values obtained.

Results:

\begin{tabular}{|l|l|l|l||}
\hline \multicolumn{1}{|l|l||}{} & 2002 pre-Bilicheck & 2009 post-Bilicheck & p value \\
\hline Total babies (population) & 5692 & 7938 & \\
\hline $\begin{array}{l}\text { Total jaundiced babies } \\
\text { with SBR equal to or } \\
\text { greater than 300 }\end{array}$ & $109(1.9 \%)$ & $98(1.2 \%)$ & 0.0017 \\
\hline Babies who had SBRs & $1029(18.1 \%)$ & $915(11.5 \%)$ & 0.000 \\
\hline Total number of SBRs & 3732 & 2957 & 0.004 \\
\hline $\begin{array}{l}\text { Tests per baby - Median } \\
\text { Range }\end{array}$ & $3(1-33)$ & $2(1-27)$ & \\
\hline
\end{tabular}

Conclusions: Since the introduction of Bilicheck, there has been a significant decrease in the number of SBR tests undertaken as well as the proportion of babies having SBR measurements.

The use of Bilicheck exposes fewer neonates of $>34$ weeks gestation to painful invasive blood taking procedures. As SBRs measurements are both time and resource consuming, Bilicheck is likely to provide a more cost effective approach to the traditional method of management of jaundice. 